\title{
Evaluation of Scanner-Based Focus Finding Methods on Rough Surfaces
}

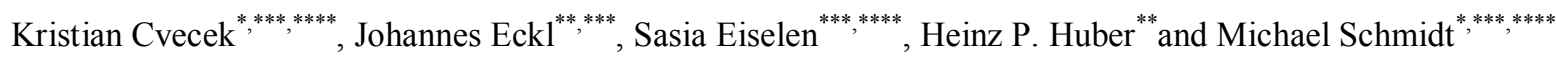 \\ * Institute of Photonic Technologies, Friedrich-Alexander-University of Erlangen-Nuremberg, \\ Konrad-Zuse-Str. 3/5, 91052 Erlangen, Germany \\ kristian.cvecek@lpt.uni-erlangen.de \\ ** Laserzentrum Hochschule München, Fakultät 06, Lothstrasse 34, 80355, Munich, Germany \\ ${ }^{* * *}$ blz-Bayerisches Laserzentrum, Konrad-Zuse Str. 2-6, 91052, Erlangen, Germany \\ ${ }^{* * * *}$ SAOT - Erlangen Graduate School in Advanced Optical Technologies, Friedrich-Alexander- \\ University of Erlangen-Nuremberg, Paul-Gordan-Str. 6, 91052 Erlangen, Germany
}

\begin{abstract}
Automated laser beam focus positioning for precise work piece alignment is highly important in laser processing. Ideally, the laser used for processing of the work piece should also be used for focus finding. For this a confocal camera can be used in front of a galvanometric scanner and objective. However, in typical laser processing applications the roughness of the sample in combination with the laser spot size and the coherence of the laser provide a confocal camera image with a distinct speckle pattern. This speckle pattern can impede the determination of the focal position with respect to the work piece, especially if simple evaluation algorithms are applied. In this work we investigate the performance of several more advanced image evaluation methods on confocal camera images for work pieces of different materials, different roughness under normal and tilted incidence of the laser beam. We show that two investigated algorithms provide a positioning uncertainty that is well below the Rayleigh length of the laser beam.
\end{abstract}

DOI: $10.2961 /$ jlmn.2015.03.0012

Keywords: focus positioning, scanner based laser processing, camera based sample alignment

\section{Introduction}

Precise automated alignment of the focal plane of the laser beam to the work piece is highly important in laser material processing. Especially, a precise focal positioning is required when ultra-short pulsed (USP) lasers are used for material modification or ablation as is implicitly (but nonetheless impressively) shown by numerous experiments investigating the USP based material ablation or modification thresholds [1-4]. In these cases nonlinear absorption mechanisms are typically exploited that depend very strongly on the laser fluence. Because USP laser based processes offer a higher potential for precise machining, any deviations from ideal focusing conditions cause a (relatively) stronger deterioration of achievable precision than laser processing mechanisms based on linear absorption.

In laser material processing exist several established distance sensing methods based on mechanical, capacitive or inductive principles. However these methods may be inadequate in terms of applicability, accuracy, ease of use or cost efficiency. Moreover, even optical methods such as optical coherence tomography [5,6] or triangulation [7] may lack in accuracy since they are insensitive to possible changes in the laser beam divergence/convergence or beam size variation. This issue can be overcome by using the processing laser beam itself (albeit attenuated) for focal plane measurements. This can be accomplished by a confocal camera that observes the back reflection of the laser beam from the sample's surfaces, comparable to the working principle of a confocal microscope [8].

While it is fairly easy to adopt this method for focus detection on plane and polished surfaces that are irradiated at normal incidence as only the brightest intensity of the back reflected spot has to be monitored, matters grow more complicated if the material surface is rough enough to provide a speckle pattern or if the irradiation occurs at tilted incidence. In the first case the confocal camera image will consist of a speckle pattern that will provide several bright spots in the image causing the simple maximum brightness evaluation method to fail. In the second case the reflected light will pass through a different region of the focusing lens on its way back to the confocal camera giving rise to possible aberrations so that the maximum brightness method may be misleading.

Unfortunately, both cases are quite common in typical laser material processing using galvanometric scanners [9] and plane field (not telecentric) focusing objectives. (For further information on use of galvanometric scanners with USP laser see [10-12] and references therein.) This is because often the samples might have a rough surface or a three-dimensional surface shape. Especially both issues occur when quasi-inline monitoring of work piece ablation is necessary to achieve three-dimensional structures $[13,14]$.

Therefore, the camera image has to be evaluated in a different way. In this work we describe four different confocal image evaluation methods starting with the rough surface of a paper sheet to provide a very distinct speckle pattern. The methods are average intensity (AI), convex hull area (CHA), speckle count (SC) and speckle size (SS). The performance of the best working methods (AI and CHA) is then applied to other sample materials with different roughnesses under normal and tilted incidence.

\section{Experimental setup}

The experimental setup schematic is shown in fig. 1 . The laser, a mode-locked Nd: $\mathrm{YVO}_{4}$ at $1064 \mathrm{~nm}$ and a pulse duration of less than $15 \mathrm{ps}$, was coupled with an expanded 
beam diameter of $6.7 \mathrm{~mm}\left(\mathrm{M}^{2}<1.5\right)$ into a galvanometer scanner and focused by a non-telecentric plane field focusing objective with a focal length of $100 \mathrm{~mm}$. This provides a focal spot diameter of $25 \mu \mathrm{m}$ and a Rayleigh length of $450 \mu \mathrm{m}$. The pulse repetition rate used in the experiments was set to $200 \mathrm{kHz}$. The laser was run at its maximum power, thus providing the best available beam profile. The power was set by using lambda-half wave-plate and a subsequent polarizer. The combination of galvanometric scanner and focusing allows to process a field of $40 \times 40 \mathrm{~mm}^{2}$ thus providing a maximum beam deflection on the sample of $\pm 20 \mathrm{~mm}$. The confocal camera is positioned behind a dielectric mirror that feeds the laser beam into the scanner system. In order to measure the focal position the laser power was reduced to power levels well below the damage threshold (in focus) of the investigated paper tissue. Nonetheless, even though the mirror in front of the galvoscanner is highly reflective for the laser wavelength, the camera's sensitivity is high enough so that the transmitted backreflected light from the sample provides sufficient signal at the camera.

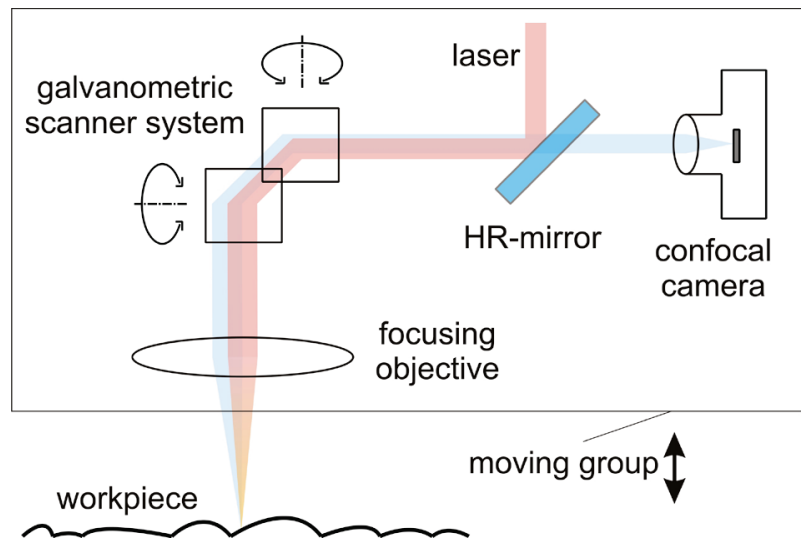

Fig. 1 Schematics of the experimental setup.

To begin with, the mechanical displacement of the focusing objective from the work piece's surface had to be found so that all further experiments could be referenced to the focal plane of the objective. To this effect the mechanical setting of the z-stage was found by structuring lines on the surface of a steel sample while stepwise displacing the moving group - consisting of the galvanometer scanner, focusing objective and confocal camera with dielectric mirror (compare fig. 1)) - from the sample's surface. The zposition of smallest achieved line-width was defined as the focal plane. In subsequent experiments the thickness of the steel sample used for this referencing as well as the different thickness of the investigated samples was taken into account.

Table 1 Surface roughness of investigated samples

\begin{tabular}{ccc}
\hline Material & $R_{q}$ in $\mathrm{nm}$ & uncertainty in $\mathrm{nm}$ \\
\hline Copper & 150 & 20 \\
Brass & 190 & 40 \\
Stainless & 410 & 20 \\
steel & & 2500 \\
Paper & 5340 & \\
\hline
\end{tabular}

Before acquiring the confocal camera images the moving group was placed so that its focal plane was positioned on the sample's surface. The laser power, camera sensitivity and exposure time were adjusted so that the recorded intensity is as high as possible but not yet saturating the camera sensor. This makes it possible to obtain usable confocal camera images for larger displacement distances from the focal plane as there is a bigger dynamic range available at the camera until the backreflected intensity drops below the dark noise level of the camera.

(a)
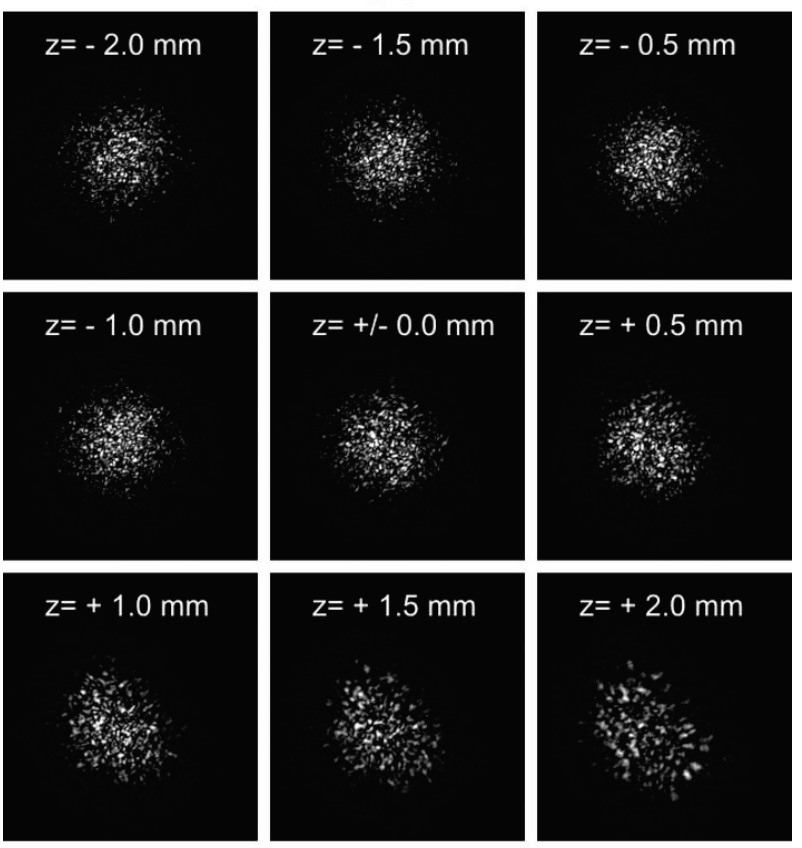

(b)
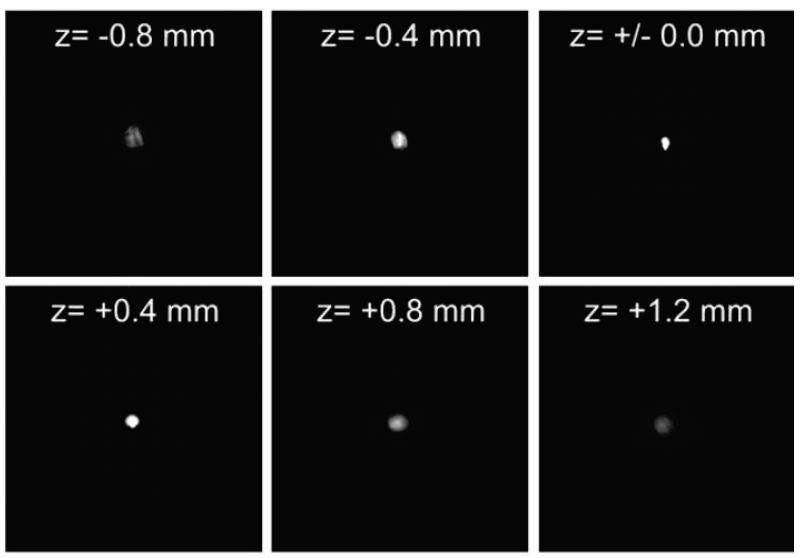

Fig. 2 Confocal camera images of paper (a) and brass (b).

In order to obtain the confocal camera images of the backreflected light from the sample's surface depending on the displacement between the sample and the moving group, the moving group was moved manually in steps of $100 \mu \mathrm{m}$ and each time a camera image was taken. For each sample and angle of incidence this procedure was repeated 5 times in order to allow for a statistical analysis of the results. The investigated angles of incidence were $0^{\circ}$ and $8.5^{\circ}$ with respect to the normal of the sample's surface. The deflection angle of $8.5^{\circ}$ corresponds to a deflection of $\pm 15 \mathrm{~mm}$ on the work piece. This was the maximum at which all samples 
yielded a usable backreflection for the confocal camera. For larger deflection angles the focusing objective and scanner were partially acting as limiting apertures.

The investigated samples were stainless steel, copper, brass and paper. The surface roughness was measured on three different locations of the sample using a confocal laser scanning microscope with a 20x magnifying objective yielding a height resolution of $10 \mathrm{~nm}$. The measured quadratic surface roughness of the samples is shown in table 1 .

Typical confocal images (normal incidence) depending on the displacement between the optics and the sample's surface are shown for paper and brass in fig. 2. It is clearly visible that the surface roughness of paper provides speckles for all displacements while the smooth surface of brass provides for most displacements only a single spot.

\section{Method description}

Of course, the method used to evaluate the focal position should be equally suitable for confocal camera images with as well as without speckles. Nonetheless, since the method to evaluate a single spot or single speckle is wellknown [8], the challenge is to find a method which can evaluate a speckle pattern. We investigated 4 different methods to evaluate the focal position that are all based upon some easily detectable properties of the confocal image's speckle pattern. These are explained as follows.

\subsection{Average intensity (AI)}

This ansatz is based on the fact that during the scan through the focal plane even a speckled image grows brighter, when averaged, the closer the focal plane of the focusing lens gets to the work piece's surface. This is due to the minimal beam diameter in the focal plane of the laser beam and, consequently, the highest intensity impinging on the surface.

This method was implemented by evaluating the pixel brightness (grey levels) of the camera image that are above a certain threshold brightness. In our case we set the threshold between 65 and 100 (out of 0-255) grey levels. This way, camera dark noise and undesirable reflections in the optical path can be excluded from evaluation. The average intensity (AI) was calculated by dividing the total sum of brightness values of all pixels above threshold by the number of pixels above threshold. A typical run of AI depending on focal displacement of the objective is shown for paper in fig. 3 .

\subsection{Convex hull area (CHA)}

This method exploits the fact, that the size of the laser beam decreases the closer the focal plane comes to the work piece's surface, even though the speckles may provide an irregularly shaped focal spot. Similar as with AI, a threshold is applied to the image to exclude noise and undesirable reflections from being evaluated. However, contrary to AI, the image is binarized using this threshold. Next, a convex hull is determined that encompasses all of the bright image regions. (The convex hull is defined as the polygon that encircles all points of a point set and has no concave indentations [12]). The area of the convex hull is calculated by counting all pixels that lie inside the convex hull region. A typical run of CHA depending on the focal displacement is depicted in fig. 3 for the paper surface.

\subsection{Speckle count (SC)}

This method exploits the property that because the beam diameter is smaller at the focal plane than outside of it, the relative roughness of a surface is smaller. Figuratively speaking, the smaller beam diameter of the waist irradiates fewer regions of different surface height thus giving a smaller number of speckles. In order to calculate the number of speckle the image is binarized using the method of Otsu [16]. Next, a median filter of $3 \times 3$ pixels is applied to the binarized image and all regions, that are at least 4connected [17], are selected and counted. The median filtering and selection of 4-connected regions is done in order to exclude eventual hot pixels of the camera, which is important especially if only few speckles are present. A typical run of the speckle count depending on focal displacement for a paper surface is shown in fig. 3 .

\subsection{Speckle size (SS)}

As can be seen in fig. 2 (a) the speckle size (SS) changes when the focal plane is displaced from the surface. The reason for this lies in the defocused imaging of the speckle pattern onto the camera image. The evaluation of this measure is similar to SC, but, instead of counting the number of regions that have connectivity of 4 or larger, the average pixel size of all more than 4-connected regions is calculated. A typical run of SS depending on focal displacement of the objective is shown for paper in fig. 3 .

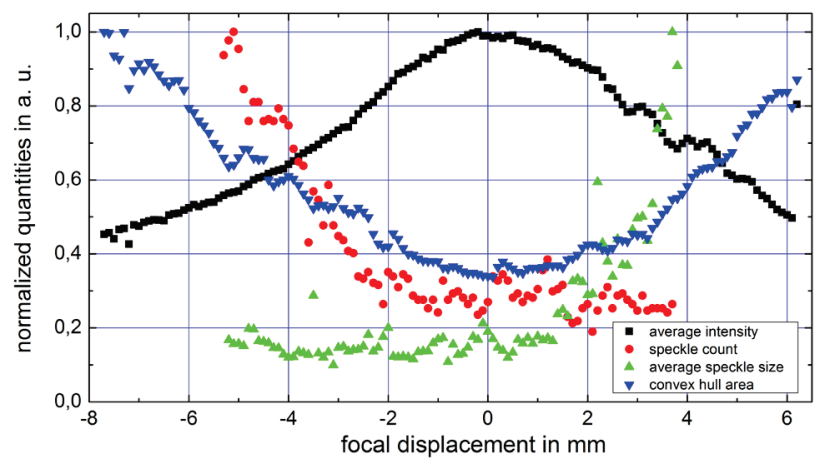

Fig. 3 Typical run of the investigated evaluation methods depending on the focal displacement for paper at normal incidence. The curves were normalized to the maximum of each investigated method.

As can be seen from fig. 3 not all methods work equally well. While AI and CHA exhibit an extremum in the vicinity of the focal spot, an unambiguous extremum is not evident for SC and SS. Since SC and SS have additionally also a longer runtime and $\mathrm{SC}$ will fail at polished surfaces that exhibit only a single spot, only the AI and CHA methods will be analyzed in the following.

\section{Evaluation of experimental results}

Fig. 4 shows the comparison between $\mathrm{AI}$ and $\mathrm{CHA}$ methods for normal and tilted incidence for all investigated samples. The values were normalized to their respective maximum in order to provide a comparable appearance. As can be seen, independently of the sample roughness or tilt angle $\mathrm{AI}$ exhibits a maximum and $\mathrm{CHA}$ a minimum in the vicinity of the focal plane. However, individual measurement points deviate from a smooth curve progression or show a large measurement uncertainty, which is more pro- 
nounced at tilted incidence (fig. 4 (b)). This is important for the determination of the correct focal position, since due to the observed fluctuations acquisition of several measurement points becomes necessary to achieve reliable results. This also necessitates the use of a best-fit curve from which the maximum (AI) or minimum (CHA) is then calculated.

From this it is clear that a steep gradient of the curve helps to determine the focal spot with a higher accuracy than a smaller gradient. As fig. 4 shows the slope of the curves is smaller for the tilted incidence than for normal incidence. Therefore a higher measurement uncertainty is to be expected for non-telecentric focusing objectives or non-flat work piece geometries.
Also the roughness has an effect on the slope of the curves. While the investigated copper, brass and steel samples had a roughness in the range of few $100 \mathrm{~nm}$ (see Table 1), which does not provide a pronounced speckle formation at $1064 \mathrm{~nm}$, the comparably high roughness of paper causes a flat slope of the curves. This in turn causes a higher positioning uncertainty.

In order to estimate the influence of roughness and tilted incidence on the measurement precision a best-fit curve was approximated and the focal displacement value of the maximum or minimum was calculated for each measurement series. The approximation was based on a Gaussian curve of the form shown in eq. 1 with $c, A, x 0$, and $\sigma$ the fit

(a)

\section{normal incidence}

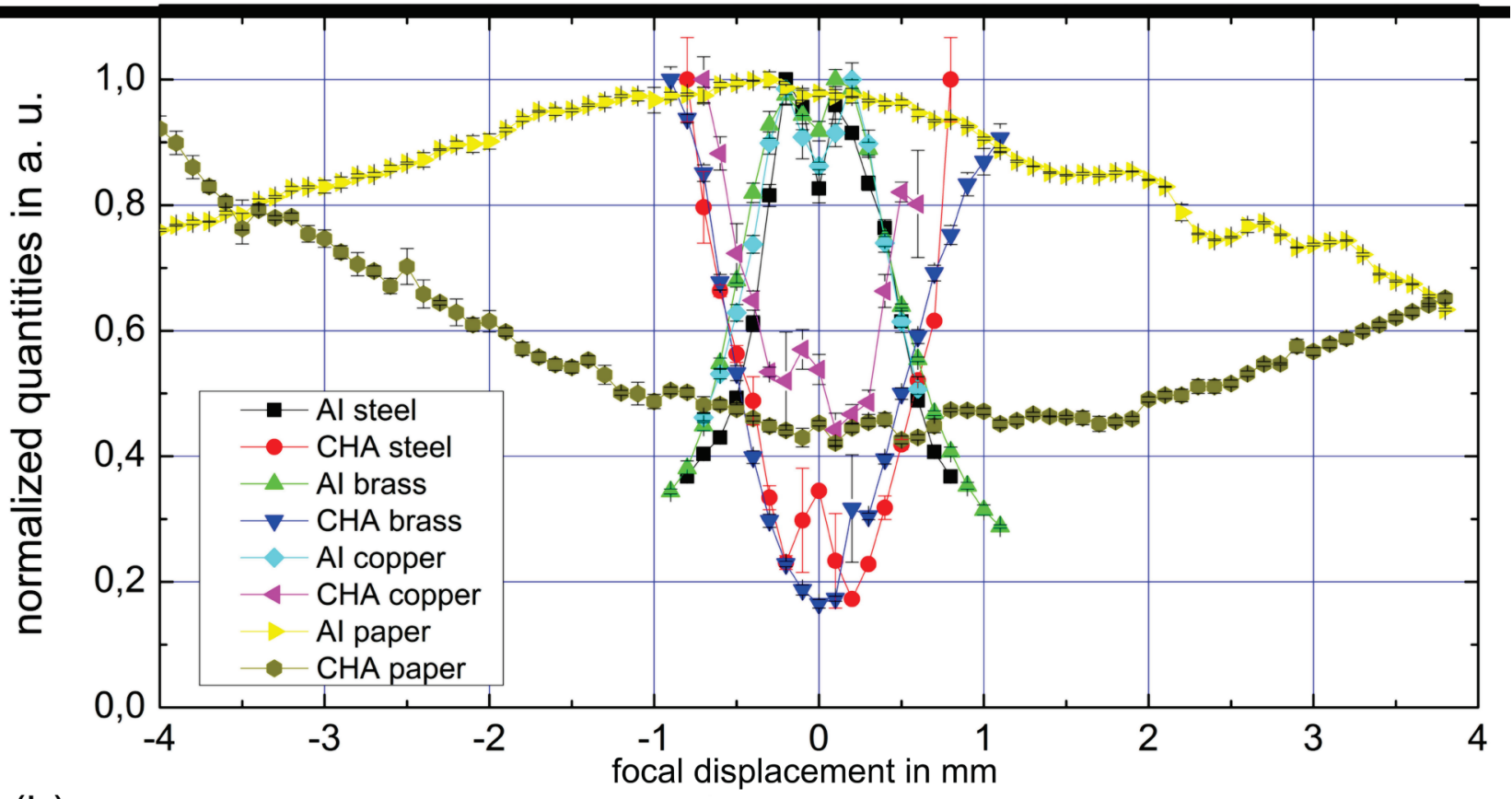

(b)

tilted incidence

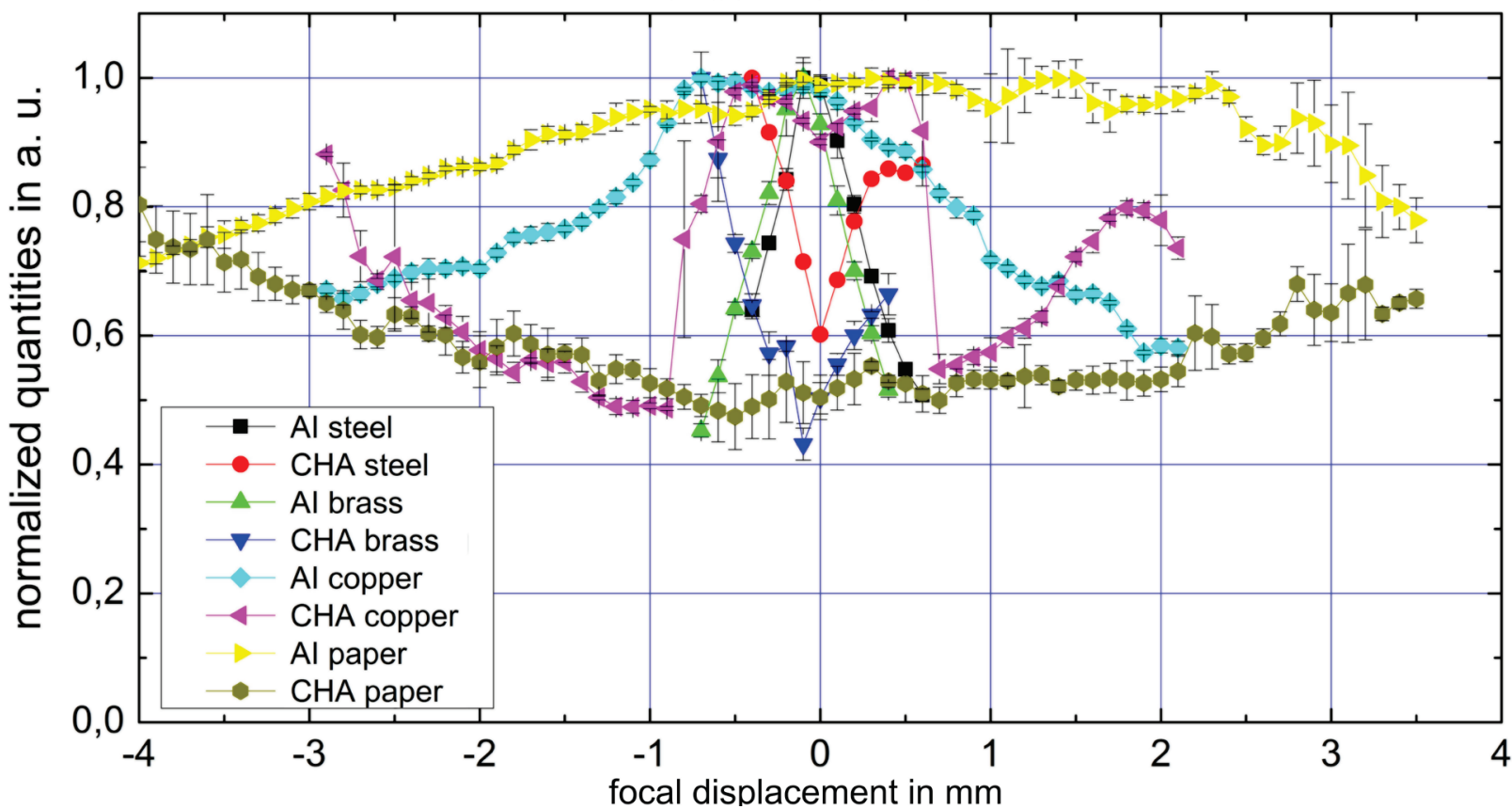

Fig. 4 Measured results for all methods and samples under normal (a) and tilted (b) incidence. 
parameters and $f$ the measurement result depending on the focal displacement $x$.

$$
f(x)=c+A \cdot \exp \left[-(x-x 0 / \sigma)^{2}\right]
$$

From the determined focal displacement $x 0$ the measurement uncertainty was calculated for each series. The uncertainty is shown in fig. 5 including the Rayleigh length of the optical system (the CHA results for copper at tilted incidence are not shown since the values increase at the focal plane and thus yield a wrong focus position).

It can be seen from fig. 5 that the reproducibility of the measurement decreases slightly for paper with its high roughness. Also the measurement uncertainty tends to be smaller for normal incidence than for tilted incidence. Nevertheless, the measurement uncertainty is smaller than the Rayleigh length of the optical system. This shows that the $\mathrm{AI}$ and CHA methods are almost equally suited (CHA slightly less) for detection of focal position independently of surface roughness or incidence angle.

Finally, the average values found for focal positions using $\mathrm{AI}$ and CHA were compared. The differences between the determined focal plane positions are shown in table 2. Disregarding the rather large difference for paper at normal incidence (and the above discussed exception for copper at tilted incidence) all other differences are below or even well below the Rayleigh length of the laser beam.

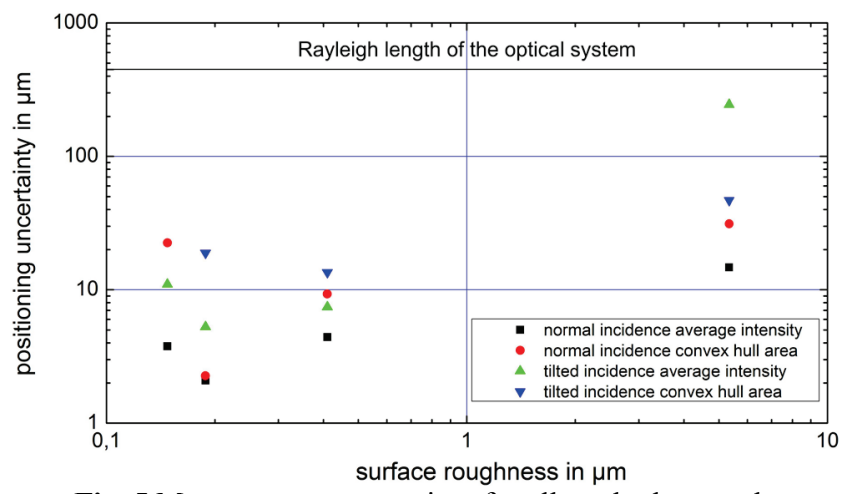

Fig. 5 Measurement uncertainty for all methods, samples and incidence conditions.

\section{Discussion}

The achieved results show for most investigated cases a good suitability for scanner based focus position detection of a rough and non-flat work piece. However, for copper under tilted incidence the CHA method has failed and there is a significant systematic difference between the focal positions found by the AI and CHA methods for paper at normal incidence. It is likely that this was caused by following reasons.

Long time laser power fluctuations (on the order of minutes or longer) may give rise to possible overexposure of sensor during the measurement run since for each surface reflectivity and roughness an optimal setting of camera parameters and laser power has to be found (see section 2). Depending on the direction of the long-term drift of the laser power the confocal camera images may get saturated or lose in brightness. In the first case the extremum of the measured curve becomes more flat and in the second case the camera images loose information due to the smaller dynamic range. Both result in a higher positioning uncertainties or even systematic errors as was the case with copper under tilted incidence (compare CHA method fig. 4 (b)) The acquired images are also affected by high frequency laser and camera noise. The process of finding the optimal

Table 2 Systematic deviation of AI and CHA methods

\begin{tabular}{ccc}
\hline Material & normal incidence & tilted incidence \\
\hline Copper & $0.022 \mathrm{~mm}$ & - \\
Brass & $0.044 \mathrm{~mm}$ & $0.088 \mathrm{~mm}$ \\
$\begin{array}{c}\text { Stainless } \\
\text { steel }\end{array}$ & $0.026 \mathrm{~mm}$ & $0.054 \mathrm{~mm}$ \\
Paper & $1.266 \mathrm{~mm}$ & $0.368 \mathrm{~mm}$ \\
\hline
\end{tabular}

camera and laser parameters as well as for the measurement procedure itself is influenced by this noise.

Although the herein described approach is suitable to show proof of concept, it is clear that the mechanical displacement of the moving group using a motorized stage is not an approach one would typically use for industrial applications where time is of the essence. Nevertheless, displacing the moving group for the measurement is not really necessary. As has been pointed out in [8], scanning the focal setting of the confocal camera's objective corresponds to moving the focusing objective. This means any auto adaptive focal system can be applied for this task with none or minimal mechanical movement which would speed up the detection of the work piece surface. Moreover, suitable systems for such focus adaptation are already commercially available and can consist of e.g. TAG lens [18], "focus shifter" [19] or focus tunable lens [20] in front of a confocal camera setup. This could speed up the detection of the focal plane up to the frame rate possible by the camera and the speed of the computer used for automated image analysis.

Another question to the broad applicability of this method in laser processing is the power level used for measurement compared to the power used processing. As it has been pointed out in section 2 the power for surface measurement should be well below the damage threshold of the material even if in focus. As it was found the highly reflective dielectric mirror in Fig. 1 transmits enough backreflected laser light, that a measurement is easily possible. Moreover, due to the same mirror the camera is in no danger to be damaged during the measurement. During processing any camera damage can be prevented by closing a mechanical shutter or aperture installed directly in front of the camera.

Nevertheless, the biggest challenge to the broad applicability of this method is the use of high power lasers where thermal lensing becomes an issue. In such a case the beam parameters will not only change during processing but will change if the laser power is set to a value below the damage threshold of the work piece for the measurement. In such a case no ideal solution exists. Either the main element which causes the thermal lensing is identified and an element such as a tilted highly reflective mirror is used to attenuate the beam, or the temporal dynamics of 
thermal lensing is exploited in such a way that the galvonometric scanner optics deflect the full power beam to beam dump near the work piece and only low energy pulses are used to measure the work piece surface with a synchronously triggered confocal camera.

\section{Conclusion and outlook}

In this work we have investigated methods to provide galvanometer-scanner based focus finding using a confocal camera setup on surfaces with different roughnesses and different angles of incidence. The acquired confocal camera images were evaluated by determining the average intensity and the convex hull area of image. Speckle based evaluation methods consisting of counting the number of speckles or determining the average speckle size failed at confocal images with distinctive speckle pattern. The average intensity and convex hull area methods were able to provide the focal position with high repeatability and an measurement uncertainty below the Rayleigh length of the laser system for almost all investigated cases.

Since the investigated methods provide an effective and non-destructive way to determine the focal position they may be useful for a wide variety of applications such as quasi in-line focus monitoring of three-dimensional structuring. However, as most of these processes have to be time efficient the measurement process must be likewise so. However, due the Gaussian-like shape of the resulting curves at least 3 points have to be measured at different focal displacements in order to allow an approximation by a best-fit curve. Likely, due to laser and camera noise, this number should be rather higher. In the current setup this would mean displacing the moving group at least 3 times during processing. It is clear that for many time relevant laser applications this is not an option.

\section{Acknowledgments and Appendixes}

This work was partially supported by the Bayerische Forschungsstiftung under the reference number AZ-111214 (project name: FoBeLas) and Photon Energy GmbH.

\section{References}

[1] J.M. Liu: Opt. Lett. 7, (1982) 196.

[2] J. Heberle, F. Klämpfl, I. Alexeev, and M. Schmidt:
J. Laser Micro/Nanoeng., 9, (2014) 103.

[3] M. Sheik-Bahae, A.A. Said, T.-H. Wei, D.J. Hagan, and E.W. van Stryland: IEEE Journal of Quantum Electronics, 26, (1990) 760.

[4] M. D. Perry, B. C. Stuart, P. S. Banks, M. D. Feit, V. Yanovsky, and A. M. Rubenchik: J. Appl. Phys., 85, (1999) 6803

[5] W. Drexler, M. Liu, A. Kumar, T. Kamali, A. Unterhuber, and R. A. Leitgeb: J. Biomed. Opt., 19, (2014) 071412.

[6] M. Wojtkowski: Appl. Opt., 49, (2010) D30.

[7] F. Blais: Journal of Electronic Imaging, 13, (2004) 231.

[8] I. Alexeev, J. Strauss, A. Gröschl, K. Cvecek, and M. Schmidt: Appl. Opt., 52, (2013) 415.

[9] G. F. Marshall and G. E. Stutz (Ed.): "Handbook of Optical and Laser Scanning," $2^{\text {nd }}$ Edition, (Publisher, CRC Press - Taylor \& Francis Publisher, 2011).

[10] O. J. Allegre, Y. Jin, W. Perrie, J. Ouyang, E. Fearon, S. P. Edwardson, and G. Dearden: Opt. Express 21, (2013) 21198.

[11]B. Neuenschwander, B. Jaeggi, M. Schmid, and G. Hennig: Proc. LANE 2014, Physics Procedia, 56, (2014) p.1047.

[12] J. Schille, L. Schneider, and U. Löschner: Appl. Phys. A, 120, (2015) 847.

[13] S. Bruening: Proc. LiM 2011, München, Germany, Physics Procedia, Volume 12, (2011) p.105.

[14] R. Schmitt: Proc. LANE 2012, Erlangen, Germany, Physics Procedia, Volume 39, (2012) p. 814.

[15]A. Erhardt: „Einführung in die Digitale Bildverarbeitung“", (Publisher, Vieweg+Teubner Verlag, 2008) p. 193.

[16] N. Otsu: IEEE Transactions on Systems, Man, and Cybernetics, 1, (1979) 62.

[17] Joseph N. Wilson and Gerhard X. Ritter: "Handbook of Computer Vision Algorithms in Image Algebra" 2nd ed., (Publisher, CRC Press, 2000) p. 177.

[18] M. Duocastella, B. Sun, and C. B. Arnold: J. Biomed. Opt., 17, (2012) 050505 .

[19] http://www.raylase.de/products/3-achsensubmodule/, retrieved on 2015/11/05.

[20] http://www.optotune.com/products/focus-tunablelenses, retrieved on 2015/11/05. 